\title{
Ultrasonographic measurement of uterine lower segment scar thickness in cases of previous one caesarean section and obstetric outcome
}

\author{
Trupthi Ganapathi, Hemangi K. Chaudhari*
}

Department of Obstetrics and Gynecology, Seth GS Medical College and KEM Hospital, Mumbai, Maharashtra, India

Received: 19 August 2018

Accepted: 27 September 2018

\section{*Correspondence:}

Dr. Hemangi K. Chaudhari,

E-mail: hemangichaudharik@gmail.com

Copyright: (C) the author(s), publisher and licensee Medip Academy. This is an open-access article distributed under the terms of the Creative Commons Attribution Non-Commercial License, which permits unrestricted non-commercial use, distribution, and reproduction in any medium, provided the original work is properly cited.

\begin{abstract}
Background: Vaginal birth after caesarean section (VBAC) has become an integral part of modern obstetrics with more than 1lakh VBACs achieved each year nationwide. Several studies have reported perinatal risks associated with failed trial of labour and uterine rupture in women attempting VBAC, due to concerns about these complications, the rate of VBAC deliveries has continued to fall in developed countries, with an inverse increase in Caesarean Sections (CS). To better assess the risk of uterine rupture, many authors have proposed sonographic measurement of scar or lower uterine segment (LUS) thickness near term, assuming that there is an inverse correlation between LUS thickness and the risk of uterine scar defect. Therefore, this assessment for the management of women with prior CS has increased safety by selecting women with the lowest risk of uterine rupture.

Methods: Present study was a prospective observational study which assessed the obstetric outcome in women with previous lower segment caesarean section willing for trial of labour. Secondly, authors aimed to ascertain the best cut off values for predicting uterine rupture.

Results: Present study found that as duration between previous LSCS and next pregnancy increased there was better chance of VBAC. As the baby weight increased VBAC rate reduced. Study also showed that scar thickness of $2.55 \mathrm{~mm}$ and above measured by transabdominal method in the third trimester can be safely given trial of VBAC.

Conclusions: Authors thus conclude that measurement of lower uterine segment/ scar thickness can help obstetrician decide whether VBAC is safe or not in patients with previous one LSCS willing for VBAC. Scar thickness of more than $2.55 \mathrm{~mm}$ can be given safe trial of labour in women with previous one lower segment caesarean section.
\end{abstract}

Keywords: Obstetric outcome, Previous one caesarean section, Ultrasonographic measurement of uterine lower segment scar thickness

\section{INTRODUCTION}

The incidence of caesarean sections is rising in many parts of the world. In 1985, WHO in consensus statement suggested that there may be no health benefits from caesarean section rates exceeding $10-15 \%$. VBAC has been advocated as a way to decrease the cost of health care by reducing the rate of caesarean delivery. In women with a single prior caesarean delivery, a trial of labour is more cost effective than an elective repeat caesarean delivery. ${ }^{1}$ Individual studies have shown achieved success rates of $72-76 \%$ for a planned VBAC after a single previous caesarean, which concurs with pooled rates derived by systematic and summative reviews. ${ }^{2-4}$

Factors influencing vaginal birth after caesarean section are:

- Success is increased in prior VBAC, appropriate counselling, sufficient personnel and equipment, non-recurrent indication, spontaneous onset of labour. 
- Failure rates are higher in increased maternal age, macrosomic fetus, multifetal pregnancy, preeclampsia, multiple prior caesarean deliveries, breech presentation, rest of non-vertex presentations, medical diseases, trans-fundal surgery, expected gestational age $>40$ weeks. $^{5}$

Birth by emergency caesarean section during the course of labour describes an unsuccessful VBAC. Maternal outcomes in an unsuccessful VBAC are uterine rupture, defined as an interruption of the uterine muscle extending to and involving the uterine serosa or interruption of the uterine muscle with further extension to the bladder or broad ligament. ${ }^{5}$ Other outcomes are hysterectomy, thromboembolism, haemorrhage, transfusion requirement, viscous injury (bowel, bladder, ureter), endometritis and maternal death.

\section{Contraindications for VBAC}

VBAC is contraindicated in women with: previous uterine rupture- risk of recurrent rupture is unknown. Previous high vertical classical caesarean section where the uterine incision has involved the whole length of the uterine corpus. ${ }^{6,7}$ Three or more previous caesarean deliveries. Women with a prior inverted $\mathrm{T}$ or $\mathrm{J}$ incision (190/10,000 rupture risk) and women with prior low vertical incision $\left(200 / 10,000\right.$ rupture risk). ${ }^{2}$ There is incomplete and disagreement information on whether the risk of uterine rupture is increased in women with previous myomectomy or prior complex uterine surgery. ${ }^{8,9}$ In fetal macrosomia and short inter-delivery interval a cautious approach is advised when considering planned VBAC in women, as there is uncertainty in the safety and efficacy of planned VBAC in such situations, the NICHD study 29 has reported a significantly decreased likelihood of successful trial of VBAC for pregnancies with infants weighing $4000 \mathrm{~g}$ or more (55$67 \%)$ compared with smaller infants (75-83\%). In the NICHD study, the rates of caesarean section in women undergoing planned VBAC were 33\%, 26\% and $19 \%$ for induced, augmented and spontaneous labour groups, respectively. ${ }^{4}$

It is difficult to precisely calculate the maternal and fetal risks associated with a trial of labour. Prenatal sonographic examination is potentially capable of diagnosing a uterine defect and determining degree of lower uterine segment thinning in patients with previous caesarean delivery. Ultrasound evaluation permits better assessment of scar complication intrapartum and could allow for safer management of delivery. Studies have shown that ultrasonography may predict uterine rupture in women with previous caesarean delivery. The risk of uterine rupture in the presence of an LSCS scar is related directly to the degree of thinning of the lower uterine segment (LUS). Transvaginal USG of uterus after a caesarean section, shows the characteristic scar formation in its anterior part.

\section{METHODS}

This is a prospective observational study conducted at a tertiary care centre in Obstetrics and Gynaecology Department between the time period of 1year, by including patients who have undergone previous one caesarean section either elective or emergency in their previous pregnancy, with no history of prior vaginal birth after caesarean section. Women registered in antenatal OPD on Thursday, fulfilling the inclusion and exclusion criteria were included in the study. Scar thickness was done for all women with previous caesarean delivery on OPD and indoor basis. Women willing for VBAC were included in the study after 36weeks gestation for measurement of lower uterine scar thickness by transabdominal sonography, women with scar thickness of more than $2 \mathrm{~mm}$ were included in the study. Ultrasound was be performed by registrars/lecturers of our Radiology Department by transabdominal method to measure scar thickness/ lower uterine segment thickness. Women was offered emergency LSCS if any indication developed during intrapartum. Intraoperative findings were noted if the women required LSCS in the present pregnancy, following delivery she was part of the study as long she required indoor management. Neonate was followed immediate postdelivery for the first 24 hours only. Written informed valid consent was taken in the language they best understand for including them in the study. Information was obtained regarding their antenatal history, investigations and intrapartum management.

\section{Inclusion criteria}

Singleton term pregnancies (after 36 weeks) with cephalic presentation with previous one caesarean section (elective or emergency) willing for VBAC.

\section{Exclusion criteria}

- Multifetal pregnancy

- Placenta previa

- Placenta accreta, increta, percreta

- Non-cephalic presentations

- More than one previous caesarean section

- Preterm labour

- Previous classical caesarean section.

\section{Parameters studied on USG}

- Thickness of lower uterine scar

- Expected weight of fetus

- Amniotic fluid Index (AFI)

- Gestational age.

\section{Maternal parameters assessed}

1. Maternal age, gravida and parity status.

2. Gestational age of termination in the present pregnancy.

3. Induced/spontaneous onset of labour. 
4. Indication for caesarean section in previous and present pregnancy.
a) Scar tenderness, maternal and fetal tachycardia
b) Prolonged labour/non-progress of labour
c) Hypertensive disorder
d) Diabetes mellitus/ gestational diabetes
e) Intrauterine Growth retardation (IUGR)
f) Bad obstetric history
g) Fetal distress
h) Failure of induction
i) Meconium stained liquor
j) Borderline pelvis
k) Antepartum haemorrhage- abruption.

5. Mode of delivery
a) VBAC
b) Caesarean section
c) Instrumental vaginal delivery.

6. Incidence of intrapartum and postpartum complications
a) Scar dehiscence
b) Uterine rupture
c) Need for blood transfusion
d) Postpartum haemorrhage
e) Need for obstetric hysterectomy
f) Perineal / postpartum haemorrhage.

\section{Perinatal parameters assessed}

- $\quad$ APGAR score at birth

- NICU admission

- Perinatal morbidity and mortality

- Birth weight of baby-its correlation with outcome of labour.

\section{RESULTS}



Figure 1: Mode of delivery in present pregnancy.

Figure 1 shows the modes of delivery in previous caesarean section women. Most of the women underwent vaginal birth after caesarean section in present study. 19 (48\%) had VBAC, $3(7 \%)$ required forceps delivery. Overall $55 \%$ delivered by vaginal mode.

Figure 2 shows outcome in a woman with previous lower segment caesarean section in present study. Duration between previous LSCS to present pregnancy when 1year, the rate of LSCS in next pregnancy was $100 \%$. When duration between previous LSCS to present pregnancy was 2 years $57 \%$ went for repeat LSCS. When duration between previous LSCS to present pregnancy was more than 3years, the repeat LSCS rate was $11 \%$. P value is significant.

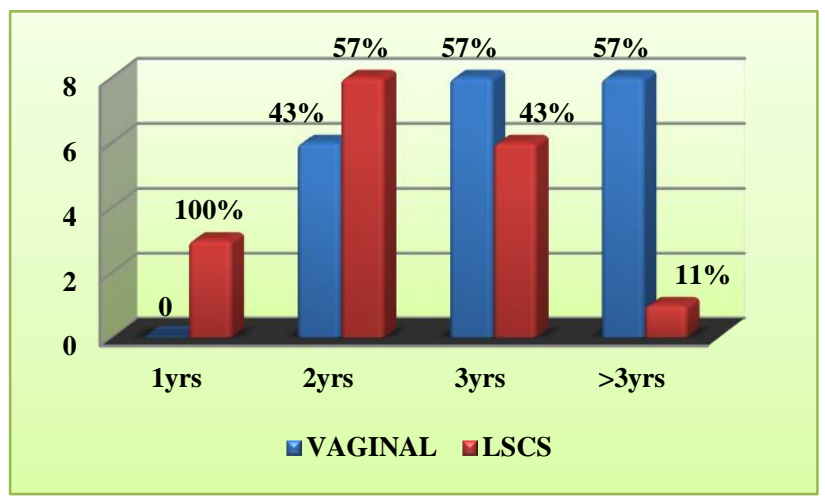

Figure 2: Duration of previous LSCS to present pregnancy affecting the outcome in present pregnancy.

As shown in figure 3 in present study, out of the 12 babies between baby weight of $2-2.5 \mathrm{~kg}, 10$ were delivered by VBAC (83.4\%) and 2 were LSCS (16.6\%). Out of the 15 babies between baby weight $2.51-3 \mathrm{~kg}, 7$ were delivered by VBAC $(46.7 \%), 8$ were by LSCS $(53.3 \%)$. And 13 babies were more than $3 \mathrm{~kg}$ of which $38.5 \%$ were delivered by VBAC and $61.5 \%$ were delivered by LSCS.

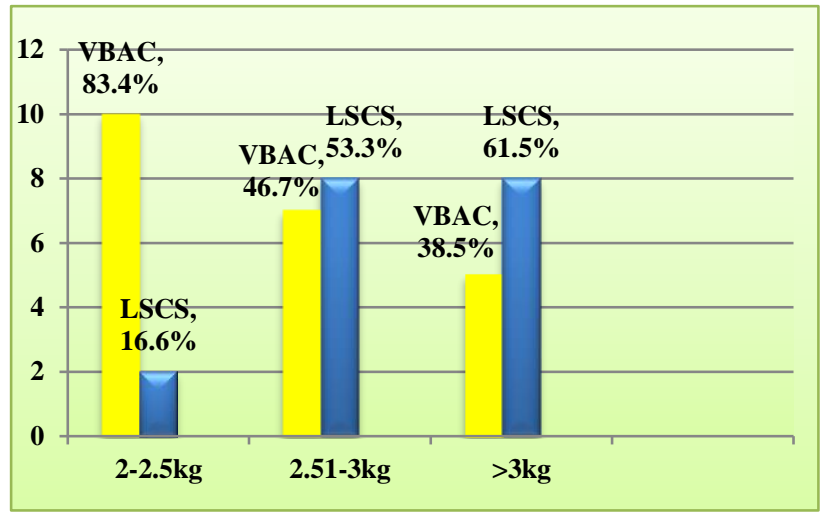

Figure 3: Weight distribution of present baby and obstetric outcome.

As shown in figure 4, present study had 22 women with scar thickness between $>2-3 \mathrm{~mm}$ of which $60 \%$ women had LSCS (13), 40\% had VBAC (9). 16 women had scar thickness between $3-4 \mathrm{~mm}$ of which $38 \%$ women had repeat LSCS (6) and 62\% women had VBAC (10). Women who had scar thickness more than $4 \mathrm{~mm}$ had $66.7 \%$ successful VBAC (2).

In present study the most common indication for LSCS was fetal distress $(44.4 \%)$. Second most common 
indication being scar tenderness $(22.22 \%)$ 3rd being meconium stained amniotic fluid $(16.67 \%)$. Maternal and fetal tachycardia are $3^{\text {rd }}$ most common $(11.11 \%)$, abruption being the least common $5.56 \%$.

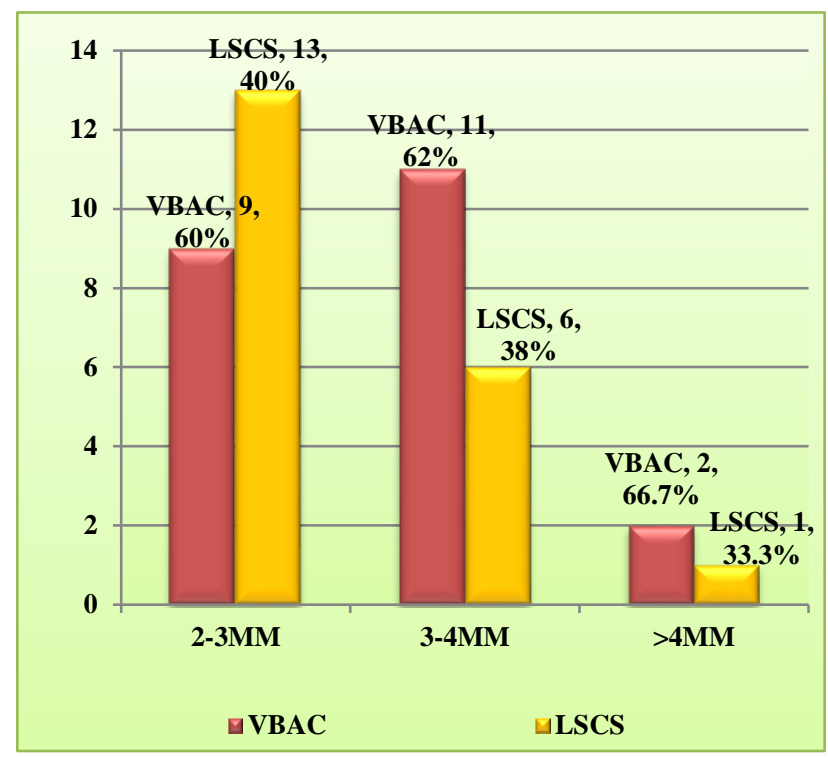

Figure 4: Scar in millimetre on transabdominal sonography and outcome.

As shown in Figure 5, present study had 2 patients of previous LSCS who were induced at term, 1 underwent LSCS and the other had VBAC. 50\% chances of induced patients resulting in repeat LSCS. P-value of this table is 0.884 indicating induction of labour or spontaneous onset of labour did not have effect on the outcome of pregnancy.

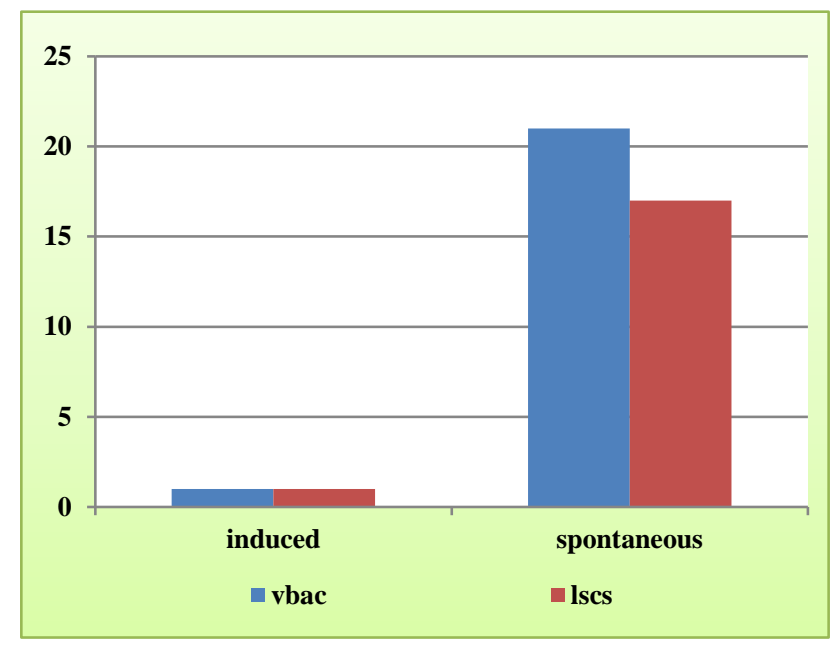

Figure 5: Mode of onset of labour and delivery outcome.

In the present study, $2.55 \mathrm{~mm}$ was considered the critical cut off value of the LUS thickness above which safe vaginal delivery can be achieved. This critical cut off value was derived from the ROC curve with sensitivity $=66.7 \%, 1$-specificity $=95.5 \%$.

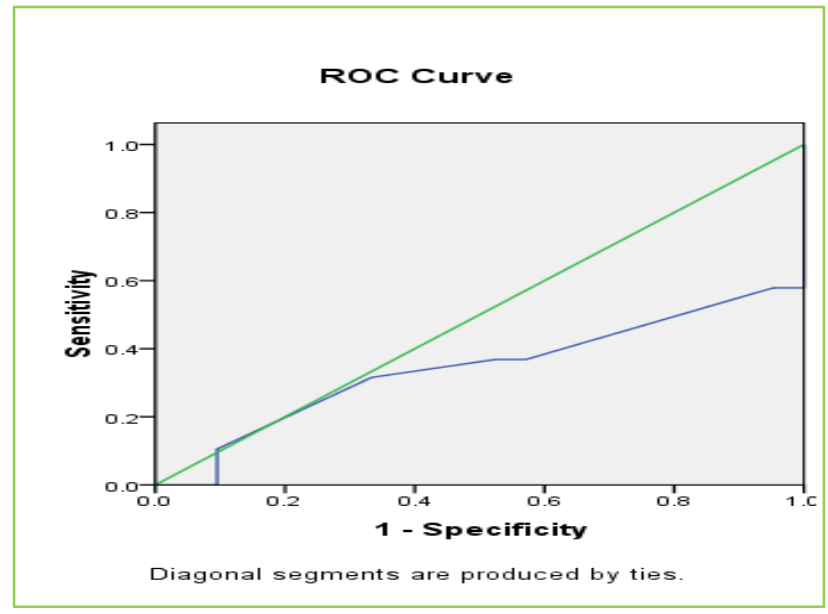

Figure 6: ROC curve predicting the scar thickness.

Table 1: Area under the curve.

\begin{tabular}{|c|c|c|c|c|}
\hline \multirow{2}{*}{ Area } & \multirow{2}{*}{$\begin{array}{l}\text { Std. } \\
\text { error }\end{array}$} & \multirow{2}{*}{$\begin{array}{l}\text { Asymptotic } \\
\text { sig. }\end{array}$} & \multicolumn{2}{|c|}{$\begin{array}{l}\text { Asymptotic } 95 \% \\
\text { confidence interval }\end{array}$} \\
\hline & & & $\begin{array}{l}\text { Lower } \\
\text { bound }\end{array}$ & $\begin{array}{l}\text { Upper } \\
\text { bound }\end{array}$ \\
\hline 0.346 & 0.090 & 0.097 & 0.169 & 0.523 \\
\hline
\end{tabular}

\section{DISCUSSION}

In this study 40 women with previous lower segment caesarean section were studied. The objective of the study was to study the association between degree of scar thickness and risk of scar dehiscence and uterine rupture. To evaluate minimum scar thickness required to prevent uterine dehiscence and scar rupture and to study the obstetric outcome of study group. The various maternal and fetal parameters were analysed and compared with standard literature.

\section{Mode of delivery in women with previous LSCS}

In present study $55 \%$ of women underwent vaginal delivery of which $7 \%$ required instrumental delivery and $45 \%$ of women required repeat caesarean delivery. In a study done by Nilanchali et al in 2014, VBAC rate in their study was $67 \% .{ }^{11}$ Another study done by Wadhwan $\mathrm{S}$ et al $63 \%$ of women with previous LSCS had successful VBAC. ${ }^{12}$ A study done by Anagha A et al in 2014 where $47 \%$ underwent successful VBAC. ${ }^{13}$ Another study done by Goel S et al in 2013 found successful VBAC in $60 \%$ of women with previous LSCS. ${ }^{13}$ In all the 4 studies mentioned including the present it is evident that successful VBAC rate varies between $50-60 \%$.

\section{Duration of previous LSCS affecting VBAC in present pregnancy}

In this study the gap in years between previous LSCS and its effect on present pregnancy was studied. Shipp et al studied the risk of scar dehiscence in relation to the interval between a previous CS and the present 
pregnancy. ${ }^{14}$ He reported that the rate of scar rupture was $2.3 \%$, when the interval was less than 18 months as compared to $1 \%$, when the interval was more than 18 months. As duration between previous LSCS and present pregnancy increases the chances uncomplicated successful VBAC increases. It has also been noticed in study by Vidhyadhar et al where the interval between a previous CS and the present pregnancy was more than two years in $77 \%, 85 \%$ cases of previous CSs delivered vaginally. ${ }^{15}$ VBAC was associated with significantly higher success rates in women whose inter-conceptional period exceeded two years $(\mathrm{P}<0.01)$ as found by Doshi et al in his study. ${ }^{16}$

\section{Outcome with respect to baby weight}

In present study out of the 12 babies between baby weight of $2-2.5 \mathrm{~kg}, 10$ were delivered by VBAC $(83.4 \%)$ and 2 were LSCS (16.6\%). Out of the 15 babies between babies between $2.51-3 \mathrm{~kg}, 7$ were delivered by VBAC (46.7\%), 8 were by LSCS (53.3\%). And 13 babies were more than $3 \mathrm{~kg}$ of which $38.5 \%$ were delivered by VBAC and $61.5 \%$ were delivered by LSCS. As the birth weight increased percentage of LSCS is found to increase. This was also found in a study by Vidhyadhar et al, birth weight of more than $3,000 \mathrm{~g}$ was associated with a lower success rate of VBAC. ${ }^{15}$ The incidence of scar dehiscence was $2 \%$ in their study. There was no maternal or neonatal mortality seen in study by Vidhyadhar B et al. ${ }^{15}$

\section{Indications for LSCS in present LSCS}

Study done by Singh $\mathrm{N}$ et al found fetal distress and meconium stained liquor were the most common indications for repeat Caesarean section similar to present study. ${ }^{11}$ Fetal distress $(13.15 \%)$ followed by scar tenderness $(5.26 \%)$ was the indication in a study done by Rajita et al for repeat LSCS. ${ }^{17}$ In a study done by Balachandra $\mathrm{L}$ et al fetal distress followed by failure to progress was the most common cause for repeat CS. ${ }^{18} \mathrm{~A}$ study done by Nikhil et al to study the indications of LSCS in previous LSCS found the most common indications of LSCS was previous LSCS. ${ }^{19}$

\section{Scar in millimeter on scan and outcome and association of scar thickness with intraoperative finding}

In a similar study done by Mohammed A et al and Sen et al, $2.5 \mathrm{~mm}$ was considered the critical cut off value of the LUS thickness above which safe vaginal delivery can be achieved below which dehiscence and rupture of uterus rate was high. ${ }^{20,21}$ Cheung et al in his another study reported that a cut off thickness of $1.5 \mathrm{~mm}$ had a sensitivity of $88.9 \%$, a specificity of $59.5 \%$, a PPV of $32.0 \%$, and a NPV of $96.2 \%$ in predicting a paper-thin or dehiscenced LUS. ${ }^{22}$ A study done by Mohammed A et al at LUS thickness $<2.5 \mathrm{~mm}$, there was a higher risk for dehiscence than those with a thickness more than 2.5 $\mathrm{mm} .{ }^{20}$ In his study he found that there is a strong correlation between degree of LUS thinning antenetally and the risk of uterine scar defect during labour. ${ }^{20}$ Thus authors can conclude that scar/LUS thickness may thus serve as an excellent predictor of uterine scar defect in women contemplating VBAC.

\section{Mode of onset of labour and obstetric outcome}

Most of previous LSCS patients willing for VBAC in present study came to the hospital with complaints of pain abdomen, leaking per vaginum or for safe confinement. Some required augmentation of labour. Most patients had successful VBAC (55\%). In 1987 Flamm et al performed a multicentre examination of 485 women who received oxytocin to augment their spontaneous labour in a planned TOL after caesarean, no increase in the risk of uterine rupture, maternal morbidity, or perinatal morbidity or mortality was detected. ${ }^{23}$ Goetzl et al examined the relation between the dose of oxytocin used and the risk of uterine rupture in women undergoing a TOL after Caesarean. ${ }^{24}$ No significant association was detected between exposure to oxytocin and the risk of uterine rupture. In 2003 Delaney and Young reported the examination of 3746 women with a prior Caesarean delivery who underwent either induced or spontaneous labour. ${ }^{25}$ They found that induced labour was associated with a greater risk of early postpartum haemorrhage, caesarean delivery, admission to a neonatal intensive care unit. There was a trend toward a higher rate of uterine rupture, but this was not statistically significant.

\section{CONCLUSION}

This is a prospective study done in a tertiary hospital in the department of Obstetrics and Gynaecology between the period August 2015-October 2016. 40 women with singleton pregnancy with cephalic presentation willing for VBAC were included in the study. Women with scar thickness more than $2 \mathrm{~mm}$ were included in the study.

Women were given TOL on consent. Emergency LSCS was performed if any indications developed during the course of labour. In present study when duration between previous LSCS and present pregnancy was less than 1 year, there was $100 \%$ rate of LSCS in next pregnancy.

When duration between previous LSCS and present pregnancy was 2 years $57 \%$ went for repeat LSCS. When duration between previous LSCS and present pregnancy was more than 3years, $11 \%$ had repeat LSCS.

Thus, authors conclude that as the inter-delivery interval increases more the chance of successful VBAC and less the rate of LSCS. When baby weight is between $2-2.5 \mathrm{~kg}$, $83.4 \%$ were delivered by VBAC between baby weight $2.51-3 \mathrm{~kg}, 46.7 \%$ were delivered by VBAC. Babies more than $3 \mathrm{~kg}, 38.5 \%$ were delivered by VBAC and $61.5 \%$ were delivered by LSCS. Thus, as the baby weight increases in a woman with previous LSCS lower the success of VBAC. The average baby weight for successful VBAC in present study was $2.69 \mathrm{~kg}$. 
In present study women with previous LSCS with scar thickness $>2-3 \mathrm{~mm}$ were 9 in number of which 5 had normal scar intra operatively $(55.5 \%)$, 3 had scar dehiscence intra operatively $(33.3 \%)$ and 1 patient had scar rupture (11.2\%). When scar thickness was between 3 $4 \mathrm{~mm}, 75 \%$ of patients had normal scar (6) and 2 had scar dehiscence $(25 \%)$ as intra operative finding. Scar thickness was more than $4 \mathrm{~mm}$ in 1 patient she had normal scar $(100 \%)$ intraoperative. Thus, it is evident that as the scar thickness on USG increased less the chance of dehiscence and rupture. In present study Spontaneous onset of labour or induction of labour had no significance in deciding whether women will have successful VBAC or repeat LSCS, $\mathrm{P}$ value is 0.6 (insignificant). Postoperative complication rate with TOL was $2.5 \%$. From our analysis and by ROC curve scar thickness of $2.55 \mathrm{~mm}$ is considered as cut off for TOL in women with previous LSCS.

\section{ACKNOWLEDGMENTS}

Authors would like to thank Professor Dr P. K. Shah for continuous support, immense knowledge and guidance during research work.

Funding: No funding sources

Conflict of interest: None declared

Ethical approval: The study was approved by the Institutional Ethics Committee

\section{REFERENCES}

1. Lavin JP, Stephens RJ, Miodovnik M, Barden TP. Vaginal delivery in patients with a prior caesarean section. Obstet Gynecol. 1982;59:135-48.

2. Landon MB, Hauth JC, Leveno KJ, Spong CY, Leindecker S, Varner MW, et al. Maternal and perinatal outcomes associated with a trial of labour after prior caesarean delivery. $\mathrm{N}$ Engl $\mathrm{J}$ Med. 2004;351:2581-9.

3. Guise JM, Berlin M, McDonagh M, Osterweil P, Chan B, Helfand M. Safety of vaginal birth after caesarean: a systematic review. Obstet Gynecol. 2004;103:420-9.

4. Chauhan SP, Martin JN, Henrichs CE, Morrison JC, Magann EF. Maternal and perinatal complications with uterine rupture in 142,075 patients who attempted vaginal birth after caesarean delivery: a review of the literature. Am J Obstet Gynecol. 2003;189:408-17.

5. Cunningham, Levono, Bloom, Spong, Dashe, Hoffman, Casey, Sheffield. Prior caesarean delivery: William's textbook of obstetrics. $24^{\text {th }}$ ed. United States: Mcgraw Hill Education; 2014:25.

6. Guise JM, Hashima J, Osterweil P. Evidence-based vaginal birth after Caesarean section. Best Prac Res Clin Obstet Gynaecol. 2005;19:117-30.

7. Turner MJ. Uterine rupture. Best Prac Res Clin Obstet Gynaecol. 2002;16:69-79.
8. Seracchioli R, Manuzzi L, Vianello F, Gualerzi B, Savelli L, Paradisi R, et al. Obstetric and delivery outcome of pregnancies achieved after laparoscopic myomectomy. Fertil Steril. 2006;86:159-65.

9. Dubuisson JB, Fauconnier A, Babaki-Fard K, Chapron C. Laparoscopic myomectomy: a current view. Human Reprod Update. 2000;6:588-94.

10. Seracchioli R, Rossi S, Govoni F, Rossi E, Venturoli $\mathrm{S}$, Bulletti C, et al. Fertility and obstetric outcome after laparoscopic myomectomy of large myomata: a randomized comparison with abdominal myomectomy. Human Reprod Update. 2000; $15: 2663-8$.

11. Singh N, Tripathi R, Mala YM. Maternal and foetal outcomes in patients with previous caesarean section undergoing trial of vaginal birth at a tertiary care centre in North India. J Preg Child Health. 2014:1:102.

12. Wadhawan S, Narone JN. Outcome of labor following previous cesarean section. Int $\mathbf{J}$ Gynecol Obstet. 1983;21(1):7-10.

13. Goel SS, Tiwari M, Hariharan C, Shrivastava DS. Outcome of post caesarean pregnancy and comparison of maternal and foetal outcome following vaginal birth versus repeat caesarean section in a rural hospital. Int $\mathrm{J}$ Reprod Contraception Obstet Gynecol. 2013;2(1):16-22.

14. Shipp TD, Zelop CM, Repke JT, Cohen A, Lieberman E. Inter delivery interval and risk of symptomatic uterine rupture. Obstet Gynecol. 2001;97:175-80.

15. Bangal VB, Giri PA, Shinde KK, Gavhane SP. Vaginal birth after cesarean section. N Am J Med Sci. 2013;5(2):140-4.

16. Doshi HU, Jain RK, Vazirani AA. Prognostic factors for successful vaginal birth after cesarean sectionAnalysis of 162 cases. J Obstet Gynecol India. 2010;60(6):498-502.

17. Jani RS, Munshi DS. Management of pregnancy with previous lower segment caesarean section in Modern obstetric practice. NHL J Med Sci. 2013;2(2):59-63.

18. Balachandran L, Vaswani PR, Mogotlane R. Pregnancy outcome in women with previous one cesarean section. J Clinic Diagnos Res: JCDR. 2014;8(2):99-102.

19. Nikhil A. Analysis of trends in LSCS rate and indications of LSCS-a study in a Medical College Hospital GMERS, Sola, Ahmedabad. Int J Pharm Bio-Sci. 2015;2(1).

20. Mohammed A, Al-Moghazi D, Hamdy M, Mohammed E. Ultrasonographic evaluation of lower uterine segment thickness in pregnant women with previous cesarean section. Middle East Fertil Soc J. 2010;15(3):188-93.

21. Sen S, Malik S, Salhan S. Ultrasonographic evaluation of lower uterine segment thickness in patients of previous caesarean section. Int J Gynaecol Obstet. 2004;87(3):215-9.

22. Cheung VY, Constantinescu OC, Ahluwalia BS. Sonographic evaluation of the lower uterine segment 
in patients with previous caesarean delivery. J Ultrasound Med. 2004;23(11):1441.

23. Flamm BL, Goings JR, Fuelbirth N, Fischermann E, Jones C, Hewson SA. Oxytocin during labor after previous Caesarean section: results of a multicenter study. Obstet Gynecol. 1987;70:709-12.

24. Goetzl L, Shipp TA, Cohen A, Zelop CM, Repke JT, Lieberman E. Oxytocin dose and the risk of uterine rupture in trial of labour after Caesarean. Obstet Gynecol. 2001;97:381-4.
25. Delaney T, Young DC. Spontaneous versus induced labor after a previous Caesarean delivery. Obstet Gynecol. 2003;102:39-44.

Cite this article as: Ganapathi T, Chaudhari HK. Ultrasonographic measurement of uterine lower segment scar thickness in cases of previous one caesarean section and obstetric outcome. Int J Reprod Contracept Obstet Gynecol 2018;7:4454-60. 